\title{
An LTCC Balanced-to-Unbalanced Extracted-Pole Bandpass Filter With Complex Load
}

\author{
Lap Kun Yeung, Member, IEEE, and Ke-Li Wu, Senior Member, IEEE
}

\begin{abstract}
In this paper, the fundamental characteristics of a novel third-order RF balanced-to-unbalanced filter, namely, a balun filter, for integrated RF module applications are presented. This center-tapped transformer-based new device works concurrently as a balun, an extracted-pole bandpass filter, and a matching network. As coupled resonant tanks are employed to perform the balun type of operation, traditional coupledresonator filter theory can thus be used to design and analyze such a new device. Moreover, an extracted-pole technique is used not only for creating a transmission zero, but also provides a capability to match the filter with a complex load. In addition to providing a simple design procedure for the device, its working mechanism is also revealed mathematically. Specifically, return-loss sensitivity with respect to each resonator admittance and complex load matching capability are discussed in details. This balun filter has been implemented in a multilayered low-temperature co-fired ceramic substrate, demonstrating its promising potentials in miniaturized RF front-end modules. Experimental measurements are also presented to validate the theory and computer simulations.
\end{abstract}

Index Terms-Balun, bandpass filter, low-temperature co-fired ceramic (LTCC), resonator filter.

\section{INTRODUCTION}

$\mathbf{T}$ HERE IS an ever increasing demand for fast, reliable, and ad hoc wireless data transfer between two or more terminal devices. Typical applications include mobile phones, personal digital assistants (PDAs), laptop computers, cameras, and printers. To satisfy such a demand, each of these devices should be equipped with an RF front-end module. In any case, miniaturization and low production cost are the two most fundamental prerequisites for these modules.

One way to miniaturize an RF front-end is to embed its passive circuitries, such as antenna, bandpass filter, balun, matching network, and interconnects, into a multilayered substrate. Lowtemperature co-fired ceramic (LTCC) technology is particularly suitable for this purpose since it can offer fine pitch lines and a multilayered architecture for embedding passive components. Another way to miniaturize a front-end is to combine the required multiple functional circuitries into one device.

Manuscript received September 6, 2005; revised January 5, 2006. This work was supported by the Innovation and Technology Fund of Hong Kong, Special Administrative Region under Grant 6901523/ITS/011/03 and by the Circuit Material Division, Heraeus Inc.

The authors are with the Department of Electronic Engineering, The Chinese University of Hong Kong, Shatin, Hong Kong (e-mail: 1kyeung @ msn.com; klwu@ee.cuhk.edu.hk).

Digital Object Identifier 10.1109/TMTT.2006.871363

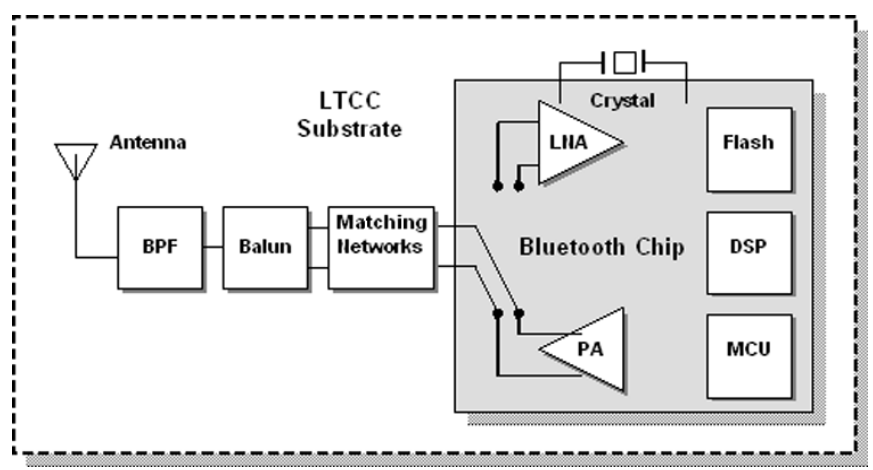

Fig. 1. Block diagram for an LTCC Bluetooth system module.

Fig. 1 shows a system block diagram of a Bluetooth module. It is seen that the three major embedded passives are matching network, bandpass filter, and balun. A balun, which converts a balanced signal to an unbalanced signal, is used in various RF circuitries such as those interfacing with RF integrated circuits (RFICs). Besides those traditionally known planar type of configurations, a number of multilayered configurations have also been recently proposed [1]-[3]. These baluns, in general, do not have any specific frequency selectivity requirements outside of its operating frequency band. While other studies [4], [5] have analyzed some topologies that offer a bandpass type of transmission response, information on how to shape up such a response has never been addressed, nor is the primary concern. Nevertheless, a drawback for all these baluns is their incapability in handling a complex load without using an additional matching network.

A new circuit architecture that combines the functionalities of a balun and a bandpass filter was recently proposed by the authors [6] together with its design procedure. In this paper, in addition to a further discussion of its design formula, the inherent property of this device that incorporates a complex load matching function is fully deliberated mathematically. With the functionalities of a matching network, a balun and bandpass filter incorporated in a single device, the size of a front-end system module, such as a Bluetooth module, can be significantly reduced. To facilitate the physical layout design of this new balun filter for high volume production and to better understand its complex loading capability, return-loss sensitivity in the passband to the parameters related to each resonator and its complex load characterization are fully studied in this paper, for the first time, by closed-form mathematical expressions. In addition, experimental verification is also presented to prove the proposed theory. 


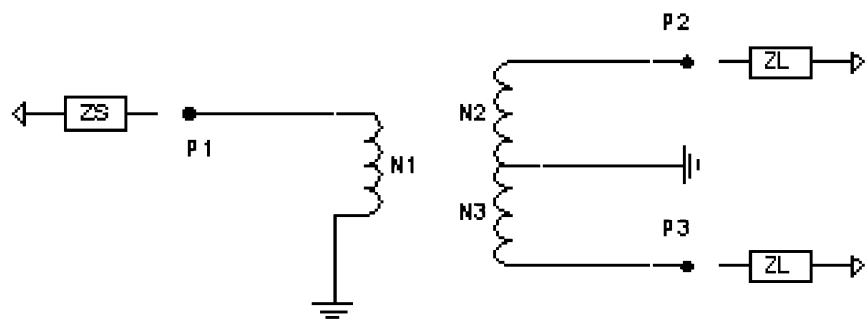

(a)

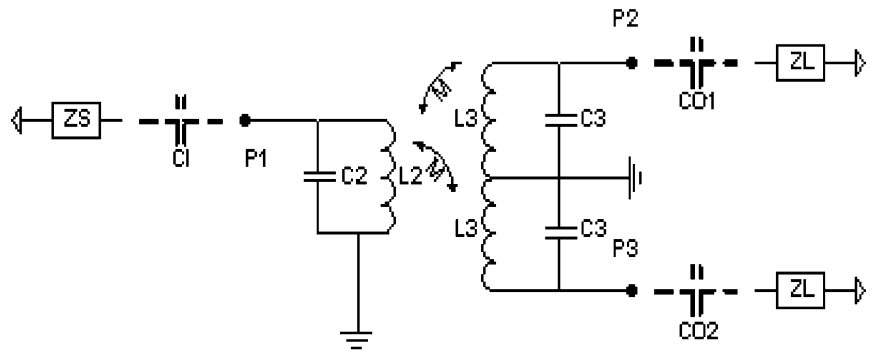

(b)

Fig. 2. (a) Ideal center-grounded transformer. (b) Proposed coupled-resonator filtering balun.

\section{THEORY}

\section{A. Fundamental Principle}

One of the fundamental representations of a balun is an ideal center-grounded transformer [7], as shown in Fig. 2(a). This transformer converts a single-ended input at port 1 to a differential output across ports 2 and 3, and vice versa, for all frequencies. In practice, however, such an ideal balun is hard to realize due to a requirement of a tight magnetic coupling and high inductance value.

Alternatively, a more practical schematic representation is shown in Fig. 2(b) where the transformer circuit is replaced by two coupled resonator tank pairs - one is positively coupled and the other is negatively coupled, where the shunted capacitors are tuned to operate at a desired frequency. As shown later, frequency dependency of this configuration can be further modified to achieve a certain type of bandpass response.

To achieve a filtering characteristic, it is helpful to view the circuit as three coupled resonant tanks. In this perspective, the conventional coupled-resonator filter theory can be used. Without losing generality, we will concentrate on the design of a third-order Chebyshev-type balun filter.

A careful inspection of Fig. 2(b) suggests that this circuit actually consists of two pairs of coupled-resonator chains. Therefore, adding an extra resonant tank and suitable input and output couplings, such as $\mathrm{CI}, \mathrm{CO} 1$, and $\mathrm{CO} 2$, would construct a balun with better bandpass properties. Fig. 3 shows our proposed third-order balun filter, which can be viewed as a merger of two third-order coupled-resonator filters with one of them having a opposite coupling between its second and third tanks. The design steps can be summarized as follows.

Step 1) Find the prototype element values for a third-order filter using the table given in [8]. An inductivetype impedance inverter should be used between the second and third tanks.

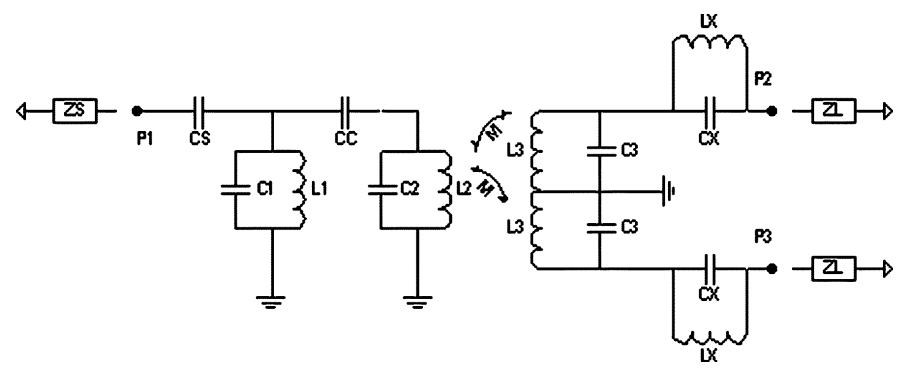

Fig. 3. Proposed third-order balun filter.

Step 2) Convert the series inductor between the second and third tanks to a mutual inductance. This can be done by the method described in [9]. The positively coupled portion of Fig. 3 is then formed.

Step 3) Add a negatively coupled tank with same component values and mutual inductance as those of the positively coupled tank in Step 2).

Step 4) Reduce the mutual inductance by a factor of $\sqrt{ } 2$.

Notice that the factor of $\sqrt{ } 2$ is obtained the following way. For an ordinary pair of coupled inductors, the equivalent input impedance looking into port 1 when port 2 is connecting to a load impedance of $Z_{L}$ is given as

$$
Z_{\text {in }}=s L_{1}-\frac{s^{2} M^{2}}{s L_{2}+Z_{L}}
$$

where $M$ is the mutual inductance between the inductor pair $L_{1}$ and $L_{2}$, and $s=j \omega$ is known as complex frequency, whereas for the circuit shown in Fig. 2(b) (ignoring $C_{2}$ and $C_{3}$ ), the equivalent input impedance looking into port 1 when ports 2 and 3 are each connected to an impedance of $Z_{L}$ is

$$
Z_{\text {in }}=s L_{1}-\frac{s^{2}\left(\sqrt{2} M_{1}\right)^{2}}{s L_{2}+Z_{L}} \text { for } L_{2}=L_{3} \text { and } M_{1}=M_{2} \text {. }
$$

Now, in order to have the same return-loss performance for these two circuits, $M_{1}$ should be equal to $M / \sqrt{ } 2$.

For a typical industrial-scientific-medical (ISM) band application, a strong rejection is required at frequency of $1.9 \mathrm{GHz}$ to minimize the interference at the global system for mobile communications (GSM) band. A parallel tank is, therefore, used at each output port to create a transmission zero at that frequency and to act as an I/O coupling. This is known as an extracted-pole technique. Consequently, the inductor used in this tank has a relatively small value when comparing to the one used in a purely inductive output coupling configuration. Equations for obtaining these component values can be derived in a similar manner as that for capacitive I/O coupling [8]. Firstly, assuming each output port has an impedance of $Z_{L}=R_{L}+j X_{L}$, a transmission zero appears at frequency $\omega_{0}$ and the passband center frequency is $\omega_{c}$. Therefore, an equivalent input admittance looking out from the positively or negatively coupled third resonators toward each corresponding output load is

$$
Y_{\mathrm{eq}}=G_{\mathrm{eq}}+j B_{\mathrm{eq}}=\frac{R_{L}}{R_{L}^{2}+X^{2}}-j \frac{X}{R_{L}^{2}+X^{2}}
$$


where

$$
X=X_{L}-\left(\omega_{c} \cdot C_{X}-\frac{1}{\omega_{c} \cdot L_{X}}\right)^{-1}
$$

Consequently, by equating $G_{\text {eq }}$ to the required $J_{n, n+1}^{2} / G_{B}$ (see [8] for the definitions), the component values are obtained as

$$
L_{X}=\frac{1-\left(\frac{\omega_{c}}{\omega_{0}}\right)^{2}}{\omega_{c}} \cdot\left( \pm \sqrt{\frac{G_{B} \cdot R_{L}}{J_{n, n+1}^{2}}-R_{L}^{2}}-X_{L}\right)
$$

and

$$
C_{X}=\frac{1}{\omega_{0}^{2} \cdot L_{X}}
$$

The sign of the square root in (5) should be chosen in a way that $L_{X}$ is a positive value. Finally, the residual shunted susceptance $B_{\text {eq }}$ should be "absorbed" by its adjacent resonator.

\section{B. Complex Load Impedance Matching}

It turns out that the parallel tanks provide another favorite feature-the capability of matching a wide range of complex loads. This feature can be studied by letting $r_{L}=G_{B} R_{L}$ and $x_{L}=G_{B} X_{L}$, where $G_{B}$ is the desired terminating conductance of a prototype filter characteristics and rewriting (5) as a family of circle equation

$$
\left(r_{L}-\frac{G_{B}^{2}}{2 J_{n, n+1}^{2}}\right)^{2}+\left[x_{L}+\frac{G_{B} \omega_{c} L_{x}}{1-\left(\frac{\omega_{c}}{\omega_{0}}\right)^{2}}\right]^{2}=\left(\frac{G_{B}^{2}}{2 J_{n, n+1}^{2}}\right)^{2}
$$

with radius $a$ and center $\left(r_{c}, x_{c}\right)$ as

$$
\left.\begin{array}{rl}
a & =\frac{G_{B}^{2}}{2 J_{n, n+1}^{2}} \\
r_{c} & =\frac{G_{B}^{2}}{2 J_{n, n+1}^{2}} \text { and } x_{c}=\frac{G_{B} \omega_{c} L_{x}}{1-\left(\frac{\omega_{c}}{\omega_{0}}\right)^{2}}
\end{array}\right\} .
$$

This equation suggests that given a fixed value of $L_{x}$, any complex load lies on a circle defined by (7) will be matched. Consequently, by considering those commonly implemented inductor values using LTCC technology, a range (a set of circles) of "matchable" complex loads can be determined. An example is shown in Fig. 4. This set of curves are generated for a filter with 0.2-dB passband ripple, center frequency of $2.45 \mathrm{GHz}$, bandwidth of $250 \mathrm{MHz}$, and transmission zero at $1.9 \mathrm{GHz}$. Notice that the values of the inductor considered are between $1-8 \mathrm{nH}$.

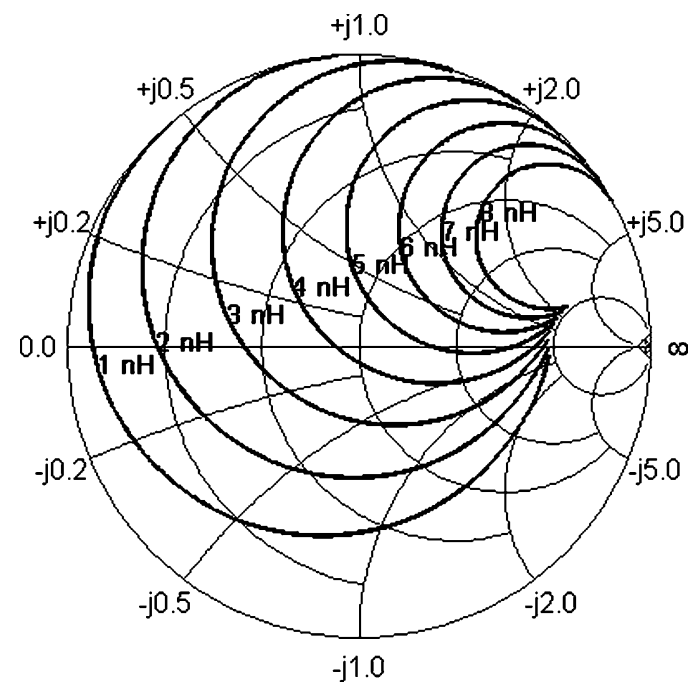

Fig. 4. Complex load matching range.

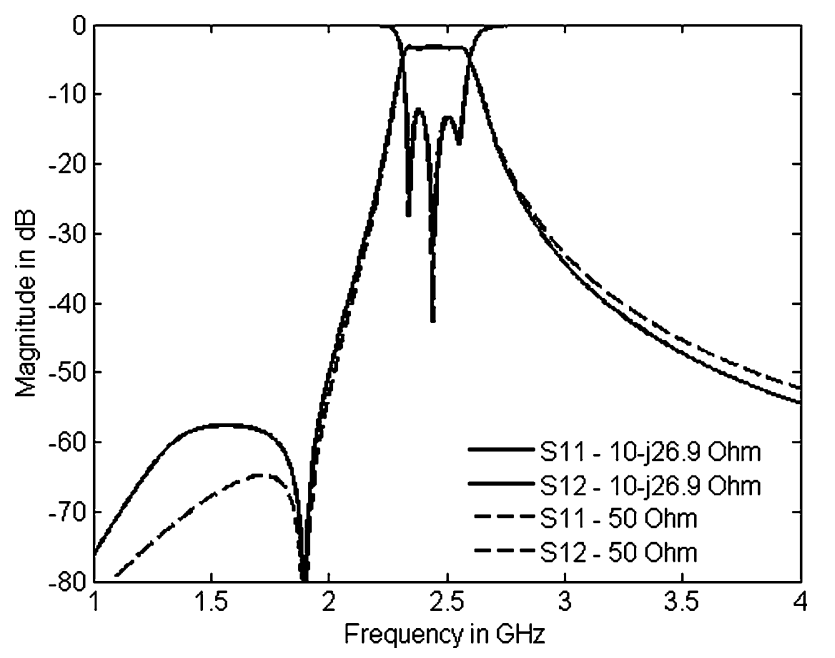

Fig. 5. Frequency responses of filter A with $10-j 26.9-\Omega$ load (solid line) and filter B with $50-\Omega$ load (dashed line).

The region spanned by the set of curves covers a large portion of the Smith chart, which means that a wide range of complex loads can be matched by using the parallel tanks with inductor values between $1-8 \mathrm{nH}$. For example, when the load impedance changes from 50 to $10-j 26.9 \Omega$, which is a typical value that we are interested in, an inductance of value $0.94 \mathrm{nH}$ is required. Frequency responses of a $10-j 26.9 \Omega$ terminated filter (A) and a standard 50- $\Omega$ terminated filter (B) are shown in Fig. 5. The corresponding component values for these two filters are listed in Table I.

\section{Return Loss Sensitivity Analysis}

As the return loss is the most sensitive parameter in a volume production, it is critical to have a better understanding of its sensitivity with regards to the circuit components. Analytical expression for the return loss of an $n$ th-order balun filter can be obtained by analyzing a generic schematic, as shown in Fig. 6 . In this figure, $J_{i, i+1}$ 's are the impedance inverters, or simply $J$-inverters, and $y_{i}$ 's are resonator admittances, whereas $y_{s}$ and $y_{L}$ 's are the source and load admittances, respectively. By the 
TABLE I

COMPONENTS FOR $10-j 26.9 \Omega$ - AND $50-\Omega$ FILTERS

\begin{tabular}{c|cc}
\multicolumn{3}{c}{ Components for 10-j26.6 Ohm and 50-Ohm filters } \\
\hline Components & Filter A & Filter B \\
\hline$C_{S}$ & $0.66 \mathrm{pF}$ & $0.66 \mathrm{pF}$ \\
$C_{C}$ & $0.27 \mathrm{pF}$ & $0.27 \mathrm{pF}$ \\
$C_{I}$ & $2.38 \mathrm{pF}$ & $2.38 \mathrm{pF}$ \\
$C_{2}$ & $2.90 \mathrm{pF}$ & $2.90 \mathrm{pF}$ \\
$C_{3}$ & $1.89 \mathrm{pF}$ & $2.65 \mathrm{pF}$ \\
$C_{X}$ & $7.50 \mathrm{pF}$ & $1.65 \mathrm{pF}$ \\
$L_{I}$ & $1.33 \mathrm{nH}$ & $1.33 \mathrm{nH}$ \\
$L_{2}$ & $1.34 \mathrm{nH}$ & $1.34 \mathrm{nH}$ \\
$L_{3}$ & $1.34 \mathrm{nH}$ & $1.34 \mathrm{nH}$ \\
$L_{X}$ & $0.94 \mathrm{nH}$ & $4.27 \mathrm{nH}$ \\
$M$ & $0.08 \mathrm{nH}$ & $0.08 \mathrm{nH}$ \\
\hline
\end{tabular}

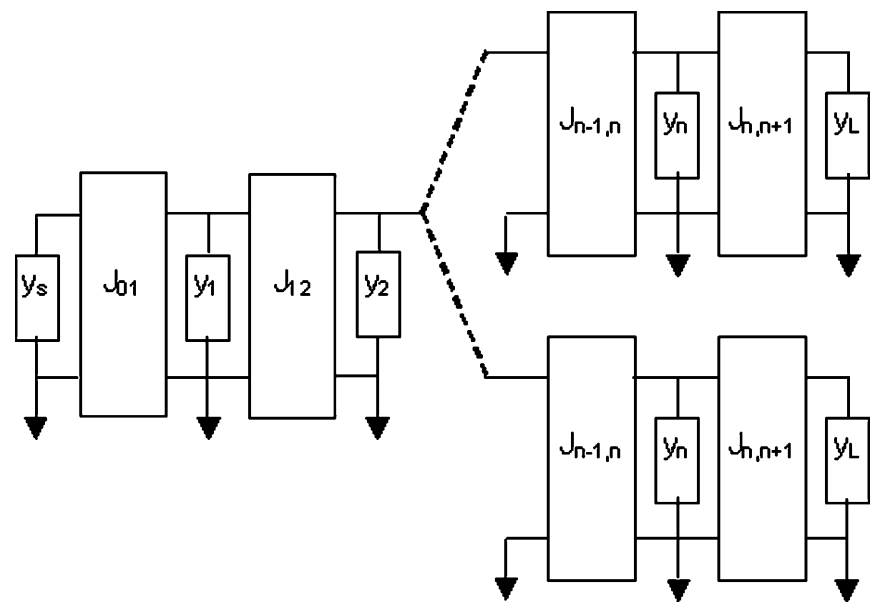

Fig. 6. Schematic diagram for an $n$ th-order balun filter.

definition of $J$-inverter, the equivalent admittance looking into $J_{01}$ can be expressed in a recursive formula as

$$
y_{1}^{\prime}=J_{01}^{2}\left(y_{1}+y_{2}^{\prime}\right)^{-1}
$$

where

$$
\left.\begin{array}{c}
y_{2}^{\prime}=J_{12}^{2}\left(y_{2}+y_{3}^{\prime}\right)^{-1} \\
y_{3}^{\prime}=J_{23}^{2}\left(y_{3}+y_{4}^{\prime}\right)^{-1} \\
\vdots \\
y_{L}^{\prime}=J_{n, n+1}^{2} y_{L}^{-1}
\end{array}\right\} \text {. }
$$

With the above equations, $s$-parameter $S_{11}$ can be calculated by substituting (9) into the following equation:

$$
S_{11}=-\frac{y_{1}^{\prime}-y_{s}}{y_{1}^{\prime}+y_{s}}=-\frac{p}{q}
$$

where $p$ and $q$ are complex polynomials.

Now, derivative of the return loss with respect to $x$, where $x$ denotes any circuit parameter, can be obtained as

$$
\frac{\partial\left|S_{11}\right|}{\partial x}=\frac{1}{|q|}\left(\frac{\partial|p|}{\partial x}-\left|S_{11}\right| \cdot \frac{\partial|q|}{\partial x}\right)
$$

This derivative is known as the un-normalized sensitivity [10]. When a filter is well tuned, the magnitude of $S_{11}$ at passband frequencies should be very small. In addition, if the second derivative term in (12) is not extremely large, which is true in most of the cases, the equation can simply be approximated as

$$
\frac{\partial\left|S_{11}\right|}{\partial x} \approx \frac{1}{|q|} \cdot \frac{\partial|p|}{\partial x} \text { for }\left|\omega-\omega_{c}\right| \leq \frac{\mathrm{BW}}{2}
$$

where BW is the filter bandwidth. Sometimes, it is necessary to have parameter $x$ normalized by its nominal value $x_{0}$ if sensitivities of different parameters are to be directly compared. In other words, the above equation should be modified to

$$
\frac{\partial\left|S_{11}\right|}{\partial \bar{x}}=x_{0} \cdot \frac{\partial\left|S_{11}\right|}{\partial x} \approx \frac{x_{0}}{|q|} \cdot \frac{\partial|p|}{\partial x} \text { for }\left|\omega-\omega_{c}\right| \leq \frac{\mathrm{BW}}{2} .
$$

Notice that this definition of sensitivity is slightly different from the usual normalized sensitivity used in other literature where (14) is further normalized by the nominal return loss value. The above equation can be written as

$$
\frac{\partial\left|S_{11}\right|}{\partial \bar{x}_{i}} \approx \frac{x_{i 0}}{|p \| q|} \cdot\left(\operatorname{Re}\{p\} \frac{\partial \operatorname{Re}\{p\}}{\partial x_{i}}+\operatorname{Im}\{p\} \frac{\partial \operatorname{Im}\{p\}}{\partial x_{i}}\right)
$$

with

$$
\frac{\partial p}{\partial x_{i}}=(-1)^{i} \frac{y_{1}^{\prime 2}}{J_{01}^{2}} \frac{y_{2}^{\prime 2}}{J_{12}^{2}} \cdots \frac{y_{i}^{\prime 2}}{J_{i-1, i}^{2}} \cdot \frac{\partial y_{i}}{\partial x_{i}}
$$

under the same condition that $\omega_{c}-\mathrm{BW} / 2 \leq \omega \leq \omega_{c}+\mathrm{BW} / 2$ and $x_{i}$ represents the $i$ th resonator reactance. In this study, we are only interested in the sensitivity relation to each resonator reactance, and un-normalized sensitivity defined in (13) is used. Notice that the same methodology can be applied to obtain the return-loss sensitivity with respect to each $J$-inverter. However, it is found that the return loss is relatively insensitive to $J$-inverters when compared to those resonators. In any case, the expression for calculating the normalized sensitivity is

$$
\frac{\partial\left|S_{11}\right|}{\partial \bar{J}_{i-1, i}} \approx \frac{J_{i-1, i}^{0}}{|p \| q|} \cdot\left(\operatorname{Re}\{p\} \frac{\partial \operatorname{Re}\{p\}}{\partial J_{i-1, i}}+\operatorname{Im}\{p\} \frac{\partial \operatorname{Im}\{p\}}{\partial J_{i-1, i}}\right)
$$

with

$$
\frac{\partial p}{\partial J_{i-1, i}}=(-1)^{i-1} \frac{y_{1}^{\prime 2}}{J_{01}^{2}} \frac{y_{2}^{\prime 2}}{J_{12}^{2}} \cdots \frac{y_{i-1}^{\prime}{ }^{2}}{J_{i-2, i-1}^{2}} \cdot \frac{2 y_{i}^{\prime}}{J_{i-1 . i}}
$$

under the same condition that $\omega_{c}-\mathrm{BW} / 2 \leq \omega \leq \omega_{c}+\mathrm{BW} / 2$.

Based on the synthesis method previously described, a $50-\Omega$ terminated third-order Chebyshev-type balanced filter of $0.2-\mathrm{dB}$ ripple, $2.45-\mathrm{GHz}$ center frequency, $250-\mathrm{MHz}$ equal-ripple bandwidth, and 1.9-GHz transmission zero has been designed. The corresponding component values of Fig. 3 are $C_{S}=0.66 \mathrm{pF}$, $C_{C}=0.27 \mathrm{pF}, C_{X}=1.65 \mathrm{pF}, L_{X}=4.27 \mathrm{nH}, C_{1}=2.38 \mathrm{pF}$, $C_{2}=2.90 \mathrm{pF}, C_{3}=2.65 \mathrm{pF}, L_{1}=1.33 \mathrm{nH}, L_{2}=1.34 \mathrm{nH}$, $L_{3}=1.34 \mathrm{nH}$, and $M=0.08 \mathrm{nH}$. Using (13) and (16), the return-loss sensitivity related to each resonator's reactance can be 


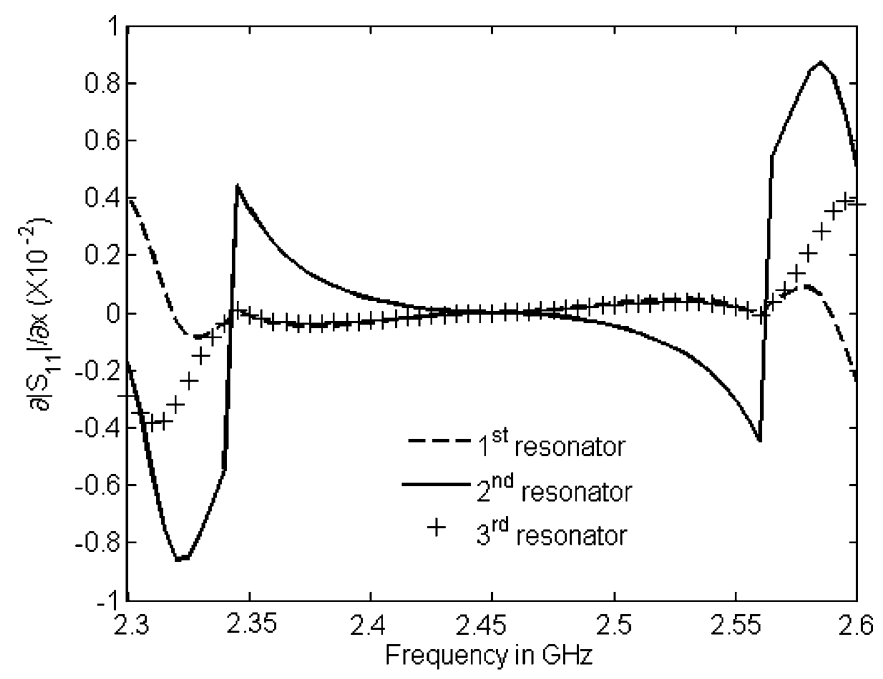

(a)

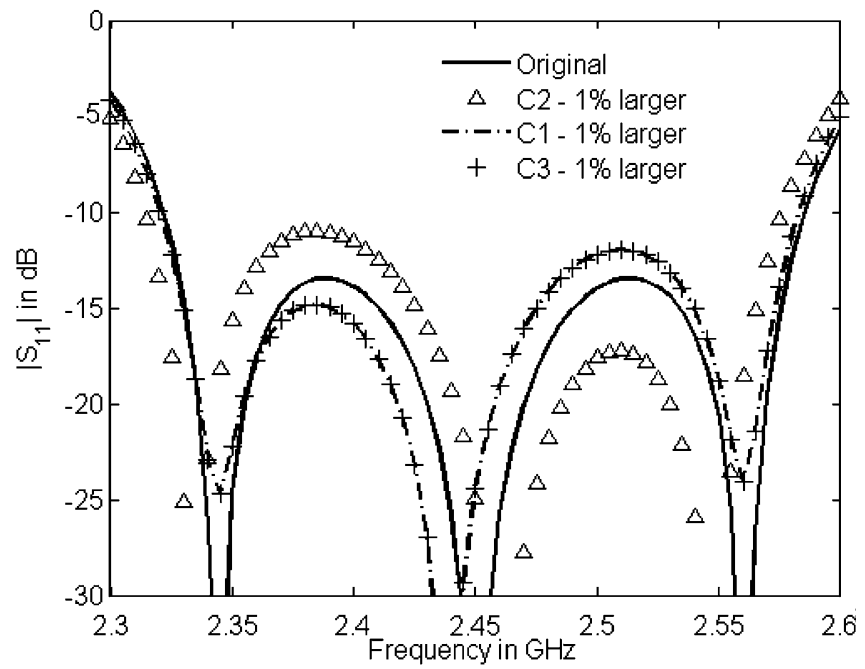

(b)

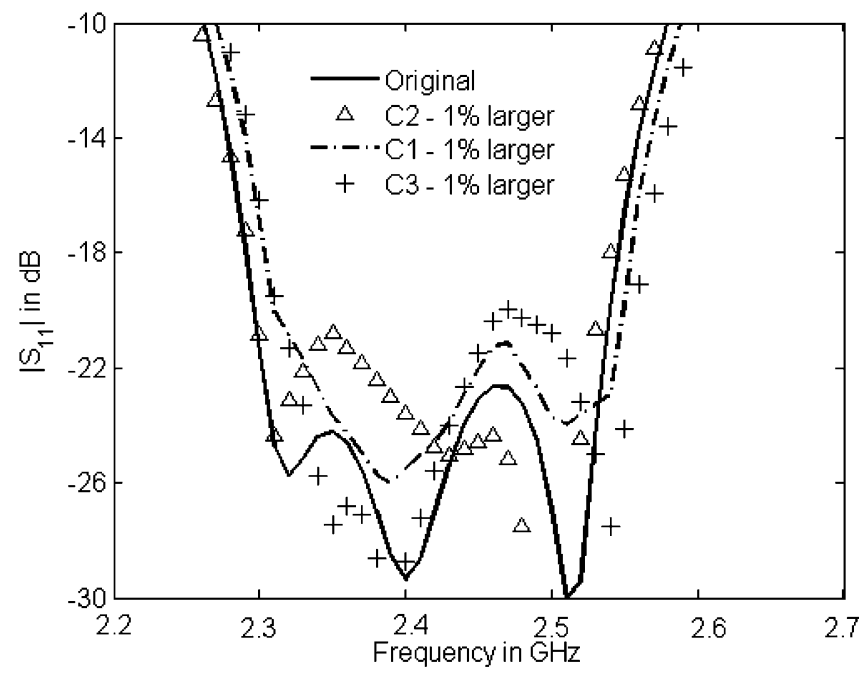

(c)

Fig. 7. (a) Return-loss sensitivity analysis for resonator impedance. (b) Changes of return loss within passband for $\pm 1 \%$ change in resonator element values. (c) Simulated return loss sensitivity analysis for $\pm 1 \%$ change in resonator element values.

calculated and they are plotted in Fig. 7(a). As seen from the results, $s$-parameter $S_{11}$ is more sensitive to the second resonator than the other two. In other words, a change on $C_{2}$ or $L_{2}$ causes a larger alteration in the return loss than any other resonator components having the same amount of changes. This property is confirmed by conducting perturbation analysis $(+1 \%$ changes in component value) on each resonator capacitor, and the results are shown in Fig. 7(b). It can be seen that with the same amount of perturbation in the component value, the change in $C_{2}$ causes a deterioration of around $3-\mathrm{dB}$ maximum change in return loss, whereas the same change in $C_{1}$ or $C_{3}$ causes only a 2-dB maximum rise. Another interesting fact is that whereas an increase in $C_{2}$ causes the return loss to rise at frequencies below the center frequency and drop at frequencies above it, an increase in any other resonator capacitor has the opposite effect. This fact can readily be seen in Fig. 7(b).

Generally speaking, the above analysis suggests that special attention should be paid to the second resonator in layout design to alleviate the sensitivity of the elements.

Fig. 7(c) shows full-wave simulated return-loss sensitivity of our layout-level balun-filter model (more details in Section III) by changing the actual physical dimensions of capacitors $C_{1}$, $C_{2}$, and $C_{3}$. Notice that this full-wave model has been further optimized for better performance. From this figure, it is seen that the return loss changes in the same trend as expected, except that $C_{3}$ has a comparable influence on the return loss as that of $C_{2}$. This is due to tight couplings between $C_{3}$ and other circuit components.

\section{PHYSICAL IMPLEMENTATION}

Having a multilayered LTCC substrate, the lumped-circuit model can be realized by using parallel plates for capacitors and printed metal strips for inductors.

As outlined in [9], an initial layout of the proposed balun filter is obtained by a very fast, but quasi-statistic electromagnetic model, and full-wave electromagnetic simulation must be carried out to predict the performance over the frequency range of interest.

Fig. 8 shows the details of a final physical layout of the proposed balun filter. It consists of a total of nine dielectric layers with each layer of approximately 3.6-mil thickness, and approximately $166 \mathrm{mil} \times 188 \mathrm{mil}$ size. Circuit elements are implemented on the bottom six layers with the first four layers mainly for capacitive elements and the other two layers for inductive elements. Notice that the transformer circuitry shown in Fig. 2(b) is simply implemented by a pair of closely spaced strips. Furthermore, a top ground plane is used to isolate the filter from other embedded components.

\section{EXPERIMENTAL RESULTS}

An experimental prototype has been built using the Heraeus HL2000 LTCC material set with a dielectric constant of approximately 7. Measurements were carried out through its three external ports located at the top of the substrate. Collected scattering parameter data was then calibrated to the desired reference planes at an internal substrate layer through the thru-reflect-line (TRL) calibration technique. The measured results together with those from a full-wave electromagnetic simulation are presented in Fig. 9. 


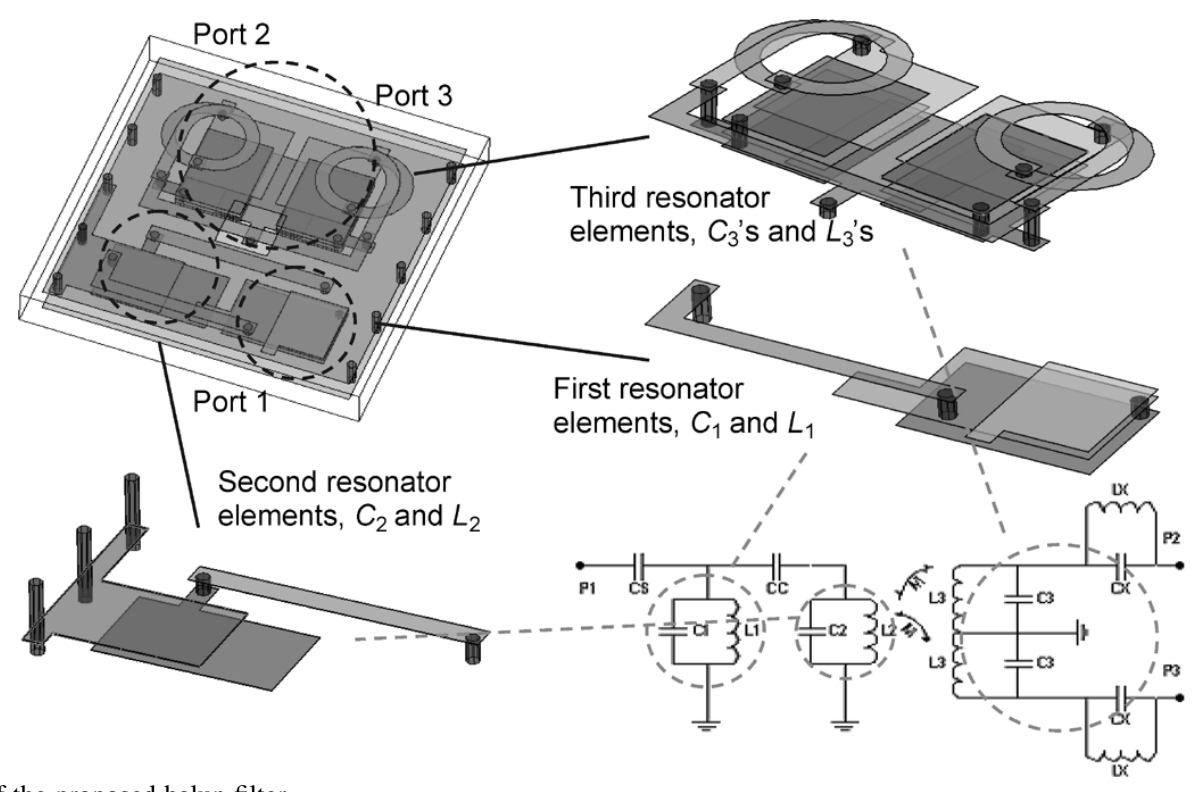

Fig. 8. Physical layout of the proposed balun filter.

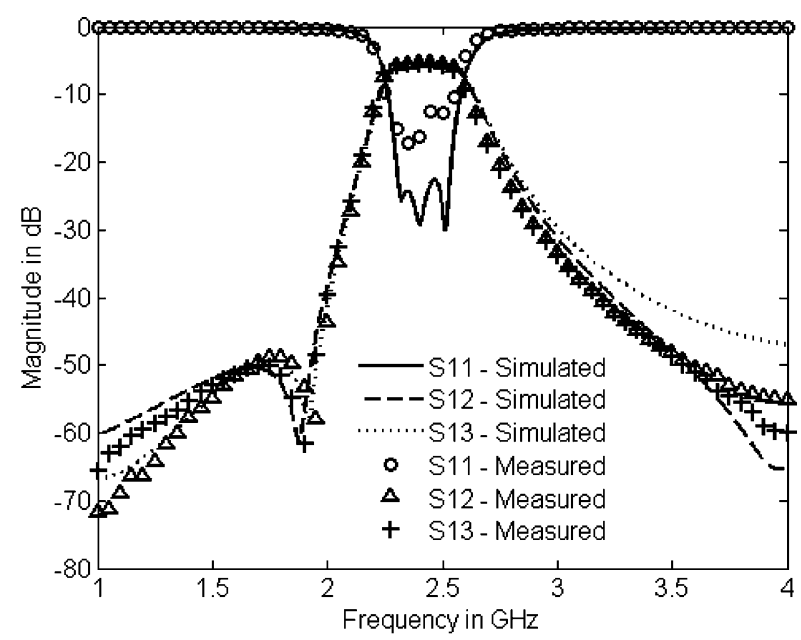

(a)

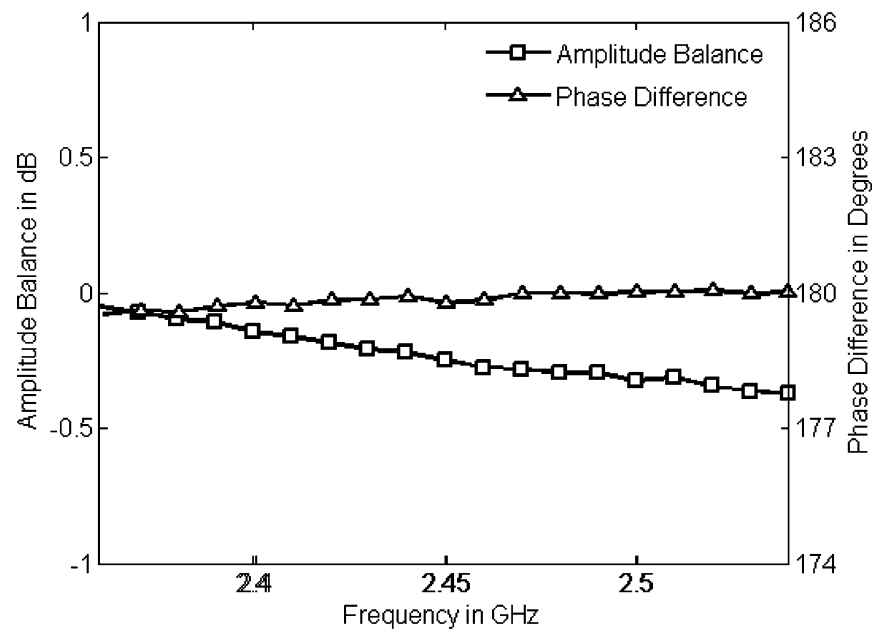

(b)

Fig. 9. Results of the balun filter. (a) Simulated and measured transmission and reflection responses. (b) Measured amplitude balance and phase difference.

A few comments about the experimental results are worth mentioning. Firstly, a deep rejection occurs, as expected, at the desired frequency of $1.9 \mathrm{GHz}$ in the transmission response. Secondly, a very good amplitude balance of approximately $0.3-\mathrm{dB}$ difference between signals at the two balanced ports is obtained. Finally, a maximum of $1^{\circ}$ phase imbalance is achieved within the passband.

However, there is a noticeable mismatch at the unbalanced port. By adjusting the circuit model shown in Fig. 3 to fit the measured responses, it is found that capacitor $C_{2}$ of the second resonator is slightly smaller than expected.

\section{CONCLUSION}

The concept of a simple multilayered passive device, which acts as a matching network, balun, and an extracted-pole bandpass filter at the same time has been presented. Its working mechanism and theory have been given mathematically. It has been shown through an experimental prototype that the balun filter exhibits a good amplitude balance, as well as a good phase balance within the operating frequency band of interest. In addition, a desired bandpass response with third-order Chebyshev-type characteristics has been achieved. To further satisfy the rejection requirement, an extracted-pole technique has been employed to sharpen the out-of-band rejection. The extracted-pole resonator tanks also play the role of an absorbing complex load. Therefore, the device also incorporates the function as a matching network. The corresponding design equations have been provided with detailed derivation. Finally, closed-form sensitivity analysis has been carried out and the results suggest that this balun filter (or a third-order filter in general) has relatively high return-loss sensitivity with respect to the second resonator elements. In general, this balun filter can serve as a good candidate for a compact LTCC RF front-end module.

\section{ACKNOWLEDGMENT}

The authors are very grateful to Circuit Material Division, Heraeus Inc., Shanghai, China, for providing the HL2000 LTCC materials for prototyping. The authors would also like to express 
their sincere gratitude to CST Ltd., Darmstadt, Germany, for providing various supports in using the CST MWS software.

\section{REFERENCES}

[1] D. W. Lew, J. S. Park, D. Ahn, N. K. Kang, C. S. Yoo, and J. B. Lim, "A design of ceramic chip balun using multilayer configuration," IEEE Trans. Microw. Theory Tech., vol. 49, no. 1, pp. 220-224, Jan. 2001.

[2] C. W. Tang and C. Y. Chang, "A semi-lumped balun fabricated by low temperature co-fired ceramic," in IEEE MTT-S Int. Microw. Symp. Dig., 2002, pp. 2201-2204.

[3] _ - "Using buried capacitor in LTCC-MLC balun," Electron. Lett., vol. 38, pp. 801-803, Jul. 2002

[4] Y. C. Leong, K. S. Ang, and C. H. Lee, "A derivation of a class of 3-port baluns from symmetrical 4-port networks," in IEEE MTT-S Int. Microw. Symp. Dig., 2002, pp. 1165-1168.

[5] K. S. Ang, Y. C. Leong, and C. H. Lee, "Analysis and design of miniaturized lumped-distributed impedance-transforming baluns," IEEE Trans. Microw. Theory Tech., vol. 51, no. 3, pp. 1009-1017, Mar. 2003.

[6] L. K. Yeung and K.-L. Wu, "An integrated RF balanced-filter with enhanced rejection characteristics," in IEEE MTT-S Int. Microw. Symp. Dig., 2005, pp. 713-716.

[7] K. V. Puglia, "Electromagnetic simulation of some common balun structures," IEEE Micro, pp. 56-61, Sep. 2002.

[8] G. L. Matthaei, L. Young, and E. M. T. Jones, Microwave Filters, Impedance Matching Networks and Coupling Structures. New York: McGraw-Hill, 1980.

[9] L. K. Yeung and K.-L. Wu, "A compact second-order LTCC bandpass filter with two finite transmission zeros," IEEE Trans. Microw. Theory Tech., vol. 51, no. 2, pp. 337-341, Feb. 2003.

[10] S. Amari, "Sensitivity analysis of coupled resonator filters," IEEE Trans. Microw. Theory Tech., vol. 47, no. 10, pp. 1017-1022, Oct. 2000.

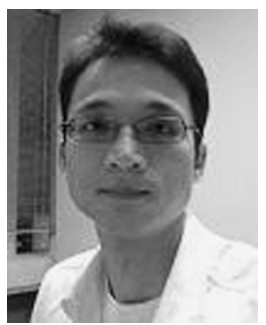

Lap Kun Yeung (S'00-M'02) received the B.Eng. degree in electrical and information engineering from the University of Sydney, Sydney, Australia, in 1998, and the M.Eng. degree in electronic engineering from the Chinese University of Hong Kong, Shatin, Hong Kong, in 2002

During 1999, he was with the Commonwealth Scientific and Industrial Research Organization (CSIRO), Sydney, Australia, where he was a Research Engineer involved in the numerical modeling of different antenna structures. He is currently with the Chinese University of Hong Kong, where he is involved in various LTCC multichip-module (MCM) designs and the development of numerical algorithms for analyzing multilayer embedded RF modules.

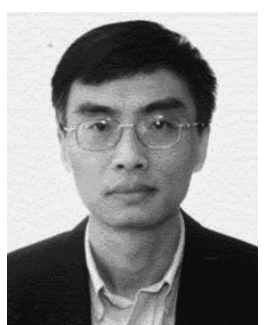

Ke-Li Wu (M'90-SM'96) received the B.S. and M.Eng. degrees from Nanjing University of Science and Technology, Nanjing, China, in 1982 and 1985, respectively, and the Ph.D. degree from Laval University, Quebec, QC, Canada, in 1989.

From 1989 to 1993, he was with the Communications Research Laboratory, McMaster University, as a Research Engineer. In March 1993, he joined the Corporate Research and Development Division, Com Dev International, where he was a Principal Member of Technical Staff in charge of developing advanced electromagnetic (EM) design software for microwave subsystems for satellite and wireless communications. Since October 1999, he has been with the Department of Electronic Engineering, The Chinese University of Hong Kong, Shatin, Hong Kong, where he is currently a Professor. He has authored or coauthored numerous publications in the areas of EM modeling and microwave and antenna engineering. His current research interests include EM modeling, microwave filters, LTCC technology, MCM technologies, antennas for wireless terminals, and active RFID systems. 